\title{
Spin textures patterned via thermally assisted magnetic Scanning Probe Lithography for magnonics
}

\author{
Daniela Petti*a $^{\text {a }}$, Edoardo Albisetti* ${ }^{\text {a,b }}$, Giacomo Sala ${ }^{\text {a }}$, Raffaele Silvani ${ }^{\mathrm{c}, \mathrm{d}}$, Marco Madami ${ }^{\mathrm{d}}$, Silvia \\ Tacchi $^{\mathrm{c}}$, Simone Finizio ${ }^{\mathrm{e}}$, Sebastian Wintz ${ }^{\mathrm{e}}$, Annalisa Caló ${ }^{\mathrm{b}}$, Xiaorui Zheng ${ }^{\mathrm{b}}$, Jörg Raabe ${ }^{\mathrm{e}}$, \\ Elisa Riedo ${ }^{\text {b,f }}$, Riccardo Bertacco ${ }^{\text {a,g }}$ \\ ${ }^{\text {a } D i p a r t i m e n t o ~ d i ~ F i s i c a, ~ P o l i t e c n i c o ~ d i ~ M i l a n o, ~ v i a ~ G . ~ C o l o m b o ~} 8120133$ Milano, Italy; ${ }^{\mathrm{b}}$ Advanced \\ Science Research Center, CUNY Graduate Center, 85 St. Nicholas Terrace, New York, 10031 NY, \\ USA; ' Istituto Officina dei Materiali del CNR (CNR-IOM), Unità di Perugia, c/o Dipartimento di \\ Fisica e Geologia, via A. Pascoli, 06123 Perugia, Italy; ${ }^{\mathrm{d}}$ Dipartimento di Fisica e Geologia, \\ Università di Perugia, Via A. Pascoli, 06123 Perugia, Italy; ${ }^{\mathrm{P}}$ Paul Scherrer Institute, \\ Forschungsstrasse 111, 5232 Villigen PSI, Switzerland; ${ }^{\mathrm{f}}$ Tandon School of Engineering, New York \\ University (NYU), MetroTech Center, Brooklyn, 11201 NY, USA; ${ }^{\mathrm{g}}$ IFN-CNR, c/o Politecnico di \\ Milano, Piazza Leonardo da Vinci, 32, 20133 Milano, Italy
}

\begin{abstract}
Magnonics represents a promising alternative to conventional electronics for the development of energy efficient computing platforms. In this context, the nanoscale engineering of spin textures is highly appealing for the development and realization of new nanomagnonic device concepts. Here, we show that reconfigurable nanopatterned spin textures can be used to manipulate spin waves. Magnetic domains and domain walls are written by thermally assisted magnetic scanning probe lithography (tam-SPL) in exchange bias systems. In such structures, we demonstrate through microfocused Brillouin Light Scattering and time resolved scanning transmission X-ray microscopy measurements, the channeling and propagation of confined spin waves. This work opens the way to the use of engineered spin-textures as building blocks of magnonics computing devices.
\end{abstract}

Keywords: Magnonics, spin-waves, scanning probe lithography, exchange bias, domain wall, Brillouin Light Scattering (BLS), scanning transmission X-ray microscopy (STXM).

\section{INTRODUCTION}

Magnonics relies on the use of spin-waves for implementing logic and computation platforms. Due to the absence of Joule heating, their vectorial and non-linear nature, spin-waves hold promises for an efficient data transport and processing. Despite in the last years many concept of spin-waves devices have been proposed ${ }^{1-5}$, the excitation, transport and manipulation of spin-waves is still a challenge. Conventionally, the control of spin-waves is achieved via physically patterning magnetic nanostructures ${ }^{6}$ such as magnonic crystals, and magnetic micro-nanowires ${ }^{7}$. However, the channeling and propagation of spin-waves in this physically patterned channels is highly inefficient, due to the need of magnetic fields for spatially shaping the magnetization profile ${ }^{8-10}$. Recently, the concept of using spin-textures to manipulate spin-waves has been proposed ${ }^{11-14}$, taking one step further in the route towards the realization of nanomagnonics devices. As a possible solution to the aforementioned problems, recently, a new approach has been proposed by some of the authors, based on the patterning of spin textures in a continuous exchange biased film via thermally-assisted magnetic Scanning Probe Lithography (tam-SPL) ${ }^{14-16}$.

*daniela.petti@polimi.it, edoardo.albisetti@polimi.it, http://nabis.fisi.polimi.it/

Spintronics XI, edited by Henri-Jean Drouhin, Jean-Eric Wegrowe, Manijeh Razeghi,

Henri Jaffès, Proc. of SPIE Vol. 10732, 107321Q - C 2018 SPIE

CCC code: $0277-786 X / 18 / \$ 18 \cdot$ doi: $10.1117 / 12.2319866$

Proc. of SPIE Vol. 10732 107321Q-1 
By scanning the heated tip on the substrate in presence of an external magnetic field, the system undergoes a localized field cooling which sets the unidirectional anisotropy arising from the exchange bias in the direction of the external field. When the magnetic field is removed, magnetic domains and domain walls are stabilized by the local exchange bias written via tam-SPL. The patterning is completely reconfigurable, since it can be erased and rewritten with a combination of heating and external field. The absence of a physical patterning, the stability of exchange bias, and the reversibility of the magnetic patterning allows for developing fully reconfigurable magnonic structures.

In this work, $90 \mathrm{deg}$ domains and $180 \mathrm{deg}$ domain walls have been patterned via tam-SPL. Through microfocused Brillouin Light Scattering ( $\mu \mathrm{BLS}$ ) and time-resolved X-ray microscopy (STXM) experiments, we show the confinement and efficient propagation of spin-waves in these micro- and nanochannels without the application of external magnetic fields. The engineering of spin textures at the nanoscale via tam-SPL opens the way to the realization of integrated nanomagnonics computing devices.

\section{SAMPLES FABRICATION}

Two different multilayers, $\mathrm{Co}_{40} \mathrm{Fe}_{40} \mathrm{~B}_{20} 5 \mathrm{~nm} / \mathrm{Ir}_{22} \mathrm{Mn}_{78} 7 \mathrm{~nm} / \mathrm{Ru} 2 \mathrm{~nm}$ (type A) and $\mathrm{Co}_{40} \mathrm{Fe}_{40} \mathrm{~B}_{20} 20 \mathrm{~nm} / \mathrm{Ir}_{22} \mathrm{Mn}_{78} 10 \mathrm{~nm}$ / Ru $2 \mathrm{~nm}$ (type B), were deposited by DC magnetron sputtering using an AJA Orion8 system with a base pressure of $5 \cdot 10^{-9}$ Torr $^{14,15}$. Type A samples were grown on $\mathrm{Si}$ substrates in order to avoid overheating during the $\mu$ BLS measurements, while type B samples were deposited on $200 \mathrm{~nm}$ thick $\mathrm{Si}_{3} \mathrm{~N}_{4}$ membranes in order to allow experiments in transmission (see paragraph 4). The $\mathrm{CoFeB} / \mathrm{IrMn}$ bilayer constitutes the exchange bias system, while the top Ru layer is used as a capping layer. In order to tailor the blocking temperature $\left(\mathrm{T}_{\mathrm{B}}\right)$ of the exchange bias in type A samples, a controlled $18.5 \%$ concentration of Ru impurities was included in the IrMn antiferromagnetic layer, as explained in Ref [17].

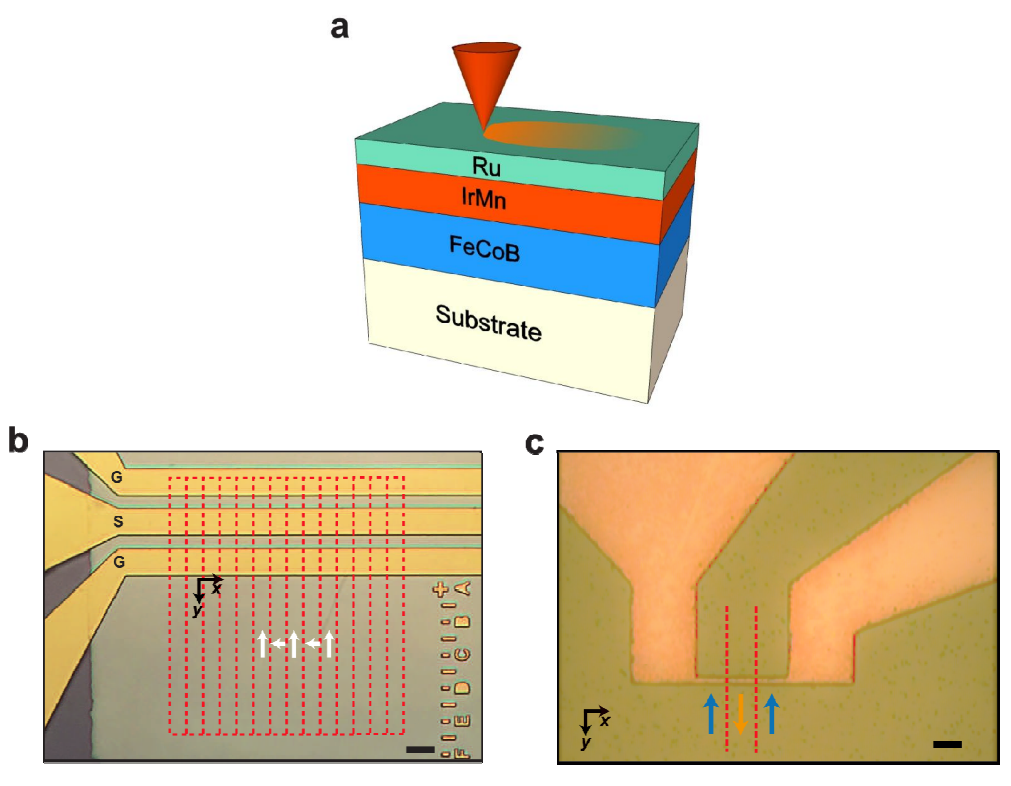

Figure 1. a. Sketch of the $\mathrm{CoFeB} / \mathrm{IrMn} / \mathrm{Ru}$ exchange bias multilayer used for patterning. The hot tip of the scanning probe microscope is used to perform a localized field cooling. b. Optical image of type A sample showing a coplanar waveguide (CPW) fabricated by EBL. The red dashed lines correspond to 90 deg domain walls separating the patterned domains. Scale bar: $5 \mu \mathrm{m}$. c. Optical image of type B sample, showing a microstrip antenna fabricated by optical lithography. The red dashed lines correspond to 180 deg domain walls patterned by tam-SPL. Scale bar: $9 \mu \mathrm{m}$. 
During the deposition, a $30 \mathrm{mT}$ magnetic field was applied in the sample plane for setting the magnetocrystalline uniaxial anisotropy direction in the $\mathrm{CoFeB}$ layer and the exchange bias direction in the as-grown sample. Then, the samples underwent an annealing in vacuum at $250{ }^{\circ} \mathrm{C}$ for 5 minutes, in a $400 \mathrm{mT}$ magnetic field oriented in the same direction as the field applied during the growth ${ }^{18}$.

Micron sized 90 deg domains were patterned on type A sample, via tam-SPL, using a customized Keysight 5600LS scanning probe system, equipped with silicon cantilevers integrated with a Joule-heating resistive heater ${ }^{19,20}$. A National Instruments DAQ NI USB-6211 in combination with MATLAB scripts and Keysight PicoView software were employed for controlling the heater temperature and the tip movement. For patterning, the thermal cantilever was raster-scanned in a zig-zag fashion in contact mode with a speed of $3 \mu \mathrm{m} \mathrm{s}^{-1}$. The separation between the lines during the scan was set to $62.5 \mathrm{~nm} .180$ deg Néel domain walls were patterned on type B sample via tam-SPL, using a NanoFrazor Explore (SwissLitho AG). The heating time was $40 \mu \mathrm{s}$ pixel $^{-1}$, with a $10 \mathrm{~nm}$ x $10 \mathrm{~nm}$ pixel size. In both cases, two rotatable permanent magnets were employed for generating a uniform external magnetic field of $60 \mathrm{mT}$ applied in the sample plane during patterning.

After the magnetic patterning, $50 \mathrm{~nm}$ thick $\mathrm{SiO}_{2}$ insulating layer was deposited by $\mathrm{RF}$ magnetron sputtering. In order to excite spin-waves, coplanar waveguides (CPW) and microstrip antenna were fabricated on samples type A and B, respectively. Coplanar waveguides with a ground to ground distance of $12 \mu \mathrm{m}$ (see Fig. 1b) were patterned using a LEO system equipped with Elphy Plus Raith pattern generator. A Ti $(7 \mathrm{~nm}) / \mathrm{Au}(100 \mathrm{~nm})$ bilayer was grown via magnetron sputtering. $2 \mu \mathrm{m} \times 30 \mu \mathrm{m}$ microstrip antennas (see Figure 1c) were fabricated via optical lithography using a Heidelberg MLA100 Maskless Aligner. A Cr $5 \mathrm{~nm} / \mathrm{Cu} 200 \mathrm{~nm}$ bilayer was deposited by means of thermal evaporation.

\section{SAMPLES CHARACTERIZATION}

The hysteresis loop of the samples after the annealing process measured by Vibrating Sample Magnetometry (VSM) are reported in Fig. 2. Both type A (panel a) and type B (panel b) samples display a sizable exchange bias of $\sim 7.5 \mathrm{mT}$ and $\sim 3$ $\mathrm{mT}$, respectively ( $\mathrm{H}_{\mathrm{E}}$ in Fig.2). The different $\mathrm{H}_{\mathrm{E}}$ in the two samples arises mainly from the different CoFeB thickness. The blocking temperature $\left(T_{B}\right)$ of the system ${ }^{17}$ for type $A$ sample, resulting from controlled inclusion of $\mathrm{Ru}$ impurities, is about $160^{\circ} \mathrm{C}$. As displayed in Fig. 2a, a field cooling from $200^{\circ} \mathrm{C}$ in $+400 \mathrm{mT}$ is therefore effective in completely shifting the initial hysteresis loop (turquoise line and dots) towards negative values (see the magenta loop), therefore reversing the exchange bias. In type B sample, due to the larger IrMn thickness and the absence of Ru impurities, $\mathrm{T}_{\mathrm{B}}$ is about $300^{\circ} \mathrm{C}$.
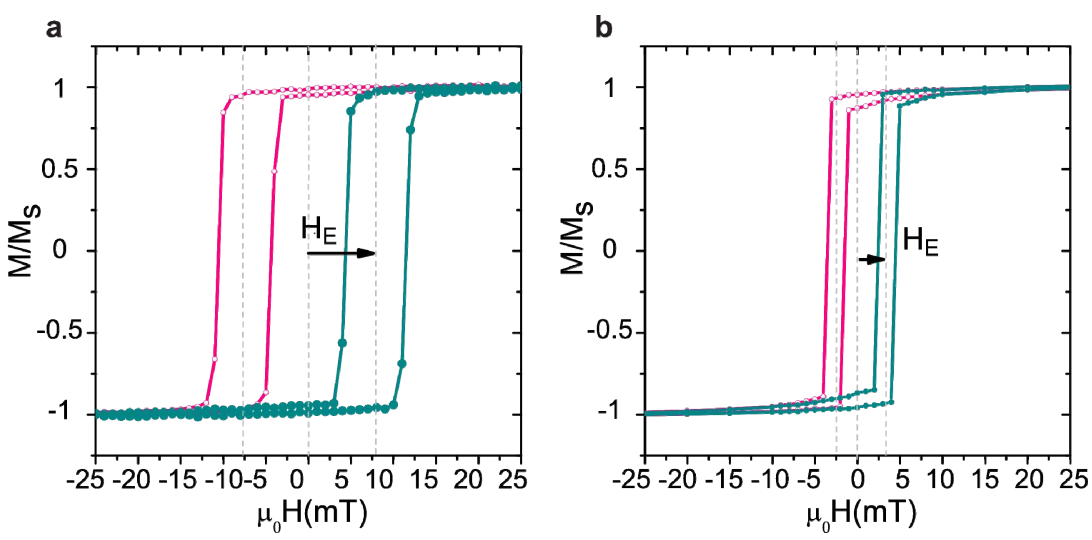

Figure 2. a. Hysteresis loops from type A sample measured after the initialization annealing at $250^{\circ} \mathrm{C}$ in $-400 \mathrm{mT}$ (turquoise line and dots) and after a field cooling from $200^{\circ} \mathrm{C}$ in $+400 \mathrm{mT}$ (magenta line and dots). b. Hysteresis loops from type $\mathrm{B}$ sample measured after the initialization annealing at $250^{\circ} \mathrm{C}$ in $-400 \mathrm{mT}$ (turquoise line and dots) and after a field cooling from $350^{\circ} \mathrm{C}$ in $+400 \mathrm{mT}$ (magenta line and dots). 
The sample is stable up to $350^{\circ} \mathrm{C}$, as the hysteresis loop measured after a field cooling from $350^{\circ} \mathrm{C}$ in $+400 \mathrm{mT}$ magnetic field demonstrates in Fig. 3b (magenta line and dots), where the exchange bias has been completely reversed without affecting its intensity. Both type A and type B samples are therefore suitable samples to perform tam-SPL, since the exchange bias and thus the unidirectional anisotropy can be controlled by performing different field coolings.

Engineered spin textures are patterned via tam-SPL as demonstrated in Fig. 3, which shows magnetic force microscopy (MFM) images of the samples at remanence. Magnetic Force Microscopy (MFM) was performed with a Keysight 5600LS system in lift mode with a $100 \mathrm{~nm}$ lift height. The MFM image of type A sample (panel a) shows 2.5 - $\mu \mathrm{m}$-wide rectangular domains with alternating 0 deg and 90 deg oriented remanent magnetization (white arrows), obtained by writing the unidirectional anisotropy along $\mathrm{x}$ and $-\mathrm{y}$, respectively. Sharp 90 deg Néel domain walls separate the domains. By patterning 180 deg domains, it is possible to stabilize straight 180 deg Néel domain walls, as displayed in the MFM image of type B sample (panel b), where the orange (blue) arrows indicate the magnetization lying along $+y(-y)$.

a

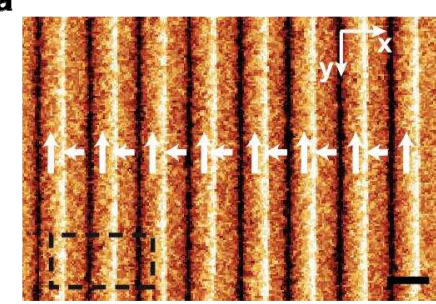

C

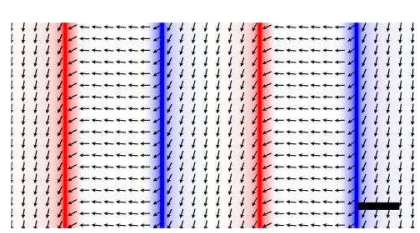

b

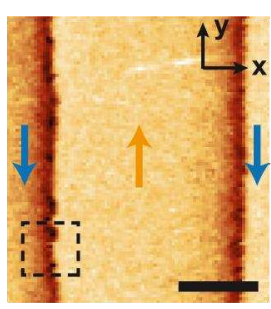

d

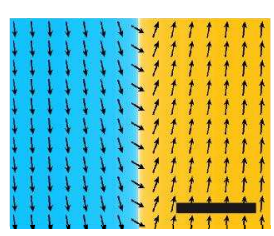

Figure 3. a. MFM image taken from type A sample. 90 deg Néel domain walls separate $2.5 \mu \mathrm{m}$ wide rectangular domains with remanent magnetization at 0 deg and 90 deg alternatively (white arrow). Scale bar: $4 \mu \mathrm{m}$. b. MFM image taken from type B sample. Two $180 \mathrm{deg}$ Néel domain walls are stabilized by patterning $180 \mathrm{deg}$ domains. The orange and blue arrows indicate the remanent magnetization. Scale bar: $5 \mu \mathrm{m}$. c. Micromagnetic simulations, showing the spin configuration of the patterns of panel a. The black arrows indicate the local magnetization direction. The red/blue colors mark the presence of magnetic charges due to the non-uniform magnetization in correspondence with the domain walls. Scale bar. $1 \mu \mathrm{m}$. d. Micromagnetic simulations of the spin configuration of the patterns of panel $b$. The black arrows indicate the local magnetization direction. The orange (blue) color code indicates the magnetization lying along $+\mathrm{y}(-\mathrm{y})$. Scale bar. $1 \mu \mathrm{m}$.

The MFM images are consistent with the simulations of the micromagnetic configuration at remanence reported in Figs. $3 \mathrm{c}, \mathrm{d}$. The simulations were performed using Object Oriented MicroMagnetic Framework (OOMMF) ${ }^{21}$ and MuMax $3^{22}$ platforms. The exchange bias was modeled as a static external field acting on the CoFeB layer. The parameters used include: $\mathrm{CoFeB}$ cell with size $10 \mathrm{~nm} \times 10 \mathrm{~nm} \times 5 \mathrm{~nm}$, saturation magnetization $\mathrm{M}_{\mathrm{s}}=1140 \mathrm{kA} / \mathrm{m}$, magneto-crystalline anisotropy $10^{3} \mathrm{~J} / \mathrm{m} 3$ along $y$, damping coefficient $\alpha=0.5$ and exchange stiffness $A_{\text {ex }}=1.2 \times 10^{-11} \mathrm{~J} / \mathrm{m}^{23}$.

In Fig. $3 \mathrm{c}$ the simulation corresponding to the pattern of panel a is reported, showing 90 deg domain walls separating the rectangular domains. The black arrows indicate the local direction of the magnetization, while the red/blue colors mark the presence of opposite magnetic charges, which can be qualitatively related to the opposite contrast observed in the MFM image. Fig. 3d displays the simulation related to the pattern of panel $b$, showing that a narrow 180 deg domain wall is stabilized. The black arrows indicate the local magnetization direction. The orange (blue) color code indicates the magnetization lying along $+\mathrm{y}(-\mathrm{y})$. 


\section{SPIN-WAVE PROPAGATION IN PATTERNED SPIN TEXTURES}

The patterned spin textures constitute micro- and nanochannels for the propagation of localized spin-waves modes.

Spin-waves confinement and propagation within the micron sized domains of type A sample was demonstrated by microfocused Brillouin Light Scattering ( $\mu$-BLS) measurements. $\mu$-BLS experiments were performed focusing about 5 $\mathrm{mW}$ of monochromatic light at $532 \mathrm{~nm}$, from a Diode-Pumped-Solid-State (DPSS) laser, with a microscope dark-field objective of large numerical aperture $(\mathrm{NA}=0.75)$ and super-long working distance $(4.7 \mathrm{~mm})^{24}$. In order to visualize the laser spot and the sample region under investigation, a coaxial viewing system was exploited, using the same objective, a collimated LED light source ( $455 \mathrm{~nm}$ wavelength), a beam expander and a CCD camera. A RF current between 2 and 10 $\mathrm{GHz}$ was injected in the coplanar waveguide through a picoprobe, in order to excite spin-waves (see Fig. 1b). During $\mu$ BLS measurements, the sample holder and the BLS spectrometer are controlled by the TFPDAS4-MICRO and TFPDAS4 software, developed at Kaiserslautern University, which provides an active stabilization of the lateral and vertical position of the sample with a precision of about $50 \mathrm{~nm}$.
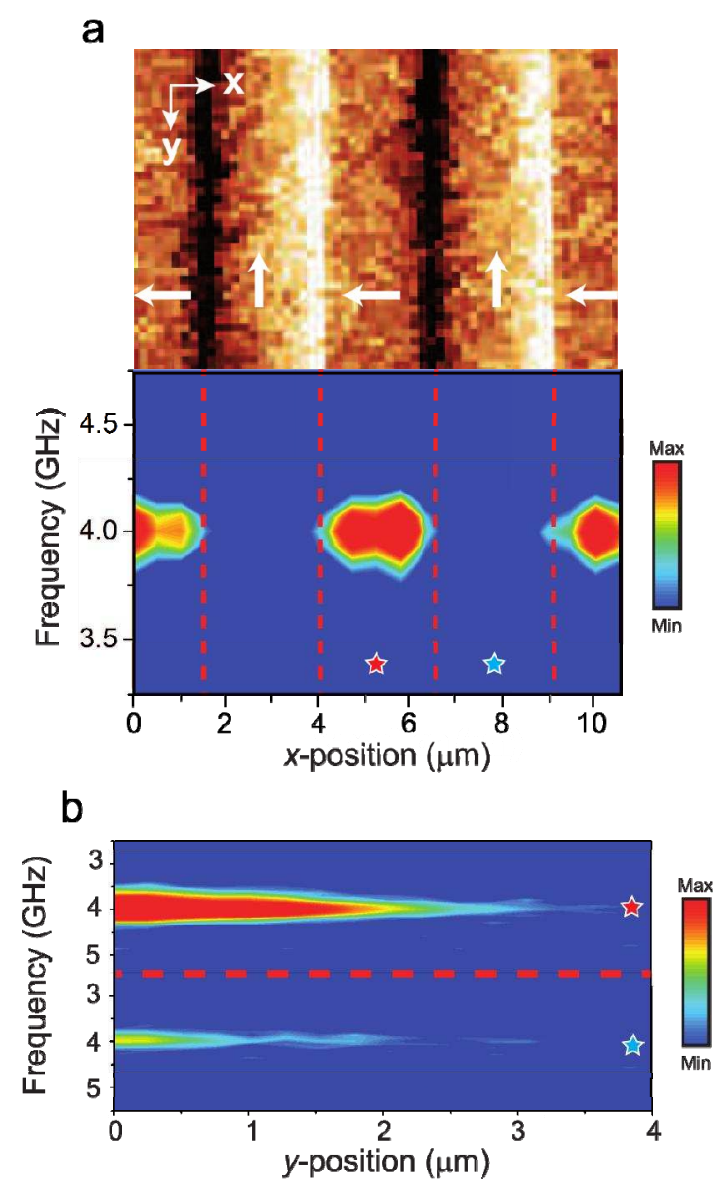

Figure 4. a. One-dimensional mapping of the spin-wave intensity measured by $\mu$-BLS along the $x$-direction, immediately outside the CPW at remanence (bottom panel). Spin-waves are efficiently excited exclusively in the domains where the static magnetization lies parallel with respect to the CPW (see the MFM zoom in the top panel). b. One-dimensional mapping of the spin-wave intensity measured along the y-direction. Spin-waves propagates efficiently only in the domain with remanent static magnetization parallel to the CPW. 
Figure 4a shows the color-coded spin-waves intensity in a logarithmic scale measured at remanence, immediately outside the $\mathrm{CPW}$, as a function of the x-position. The red dashed lines corresponds to the domains boundaries, as can be evinced by the MFM image of the top panel. It is worth to mention that, with this CPW configuration, spin-waves are efficiently excited exclusively by the coupling of the in plane dynamical component of the magnetization and the in plane Oersted field generated by the CPW. This coupling happens only in the patterned domains where the static magnetization lies parallel with respect to the CPW (white arrow in the MFM image of the top panel). Moreover, only in these domains (red star in panels a and b) spin-waves propagate efficiently, while in the others (blue star in panels a and b) they rapidly attenuate. The patterned domains represent therefore microchannels for spin-waves. Spin-waves propagates for more than $2.5 \mu \mathrm{m}$ as can be observed in Fig. 4b, which shows the color-coded spin-waves intensity as a function of the distance from the CPW (y-position).

Spin-waves propagation in the domain walls patterned on type B sample has been measured ${ }^{25}$ with spatial and temporal resolution by scanning transmission X-ray microscopy (STXM) at the PolLux (X07DA) endstation of the Swiss Light Source $^{26}$. Monochromatic X-rays, tuned to the Co L3 absorption edge (photon energy of about $781 \mathrm{eV}$ ), are focused using an Au Fresnel zone plate onto a spot on the sample, and the transmitted photons are recorded using an avalanche photodiode. The sample is scanned using a piezoelectric stage, and the transmitted X-ray intensity is recorded for each step corresponding to a pixel of the image. Magnetic contrast arises from the X-ray magnetic circular dichroism (XMCD) effect. In order to be sensitive to the in-plane component of the sample magnetization $\mathrm{M}_{\mathrm{y}}$, the samples were mounted with the surface oriented at $30 \mathrm{deg}$ with respect to the incident X-rays, as the XMCD effect probes the component of the magnetization parallel to the wave vector of the radiation. The time-resolved images were acquired stroboscopically, using an RF magnetic field generated by injecting an RF current in a microstrip antenna. The pumping signal of the injected current was synchronized to the $500 \mathrm{MHz}$ master clock of the synchrotron light source (probe signal) through a field programmable gate array (FPGA) setup. In the specific condition of the present work, the phase resolution in the time-resolved images was about $15 \mathrm{deg}$. The temporal resolution depends on the RF frequency, with its lower limit given by the width of the X-ray pulses generated by the light source (i.e. about $70 \mathrm{ps}$ FWHM).

a
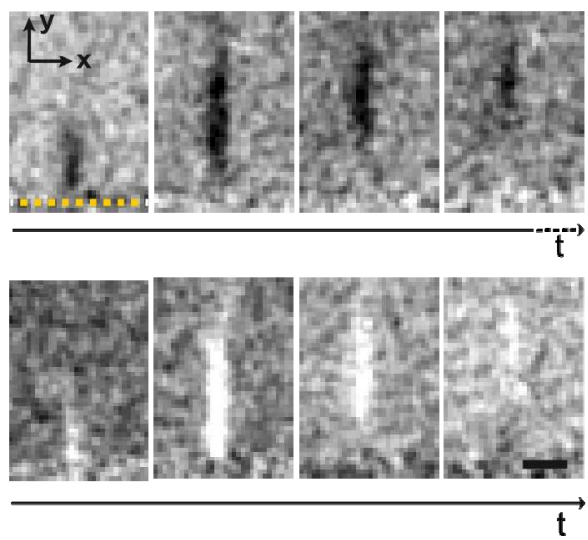

b

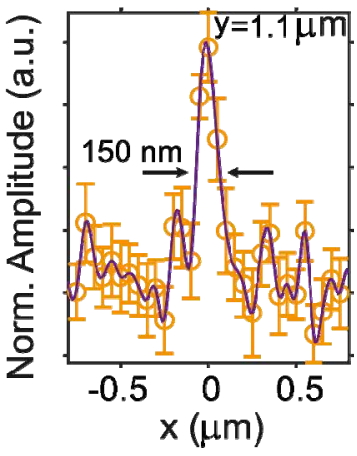

Figure 5. a. Normalized time-resolved STXM images showing the propagation of localized spin-waves along a 180 deg Néel domain wall. The frequency of the current injected in the microstrip antenna (yellow dashed line) was $0.95 \mathrm{GHz}$. Scale bar: $500 \mathrm{~nm}$. b. Horizontal profile extracted from panels a at $\mathrm{y}=1.1 \mu \mathrm{m}$ from the antenna. The FWHM of the excitation peak in correspondence of the domain wall is $150 \mathrm{~nm}$.

Figure 5 displays snapshots of the normalized $\mathrm{M}_{\mathrm{y}}$ contrast in correspondence of the 180 deg Néel domain wall of type A sample, calculated as the magnetic deviation $\Delta \mathrm{M}_{\mathrm{y}}(\mathrm{t})$ from the time-averaged state $\left\langle\mathrm{M}_{\mathrm{y}}(\mathrm{t})\right\rangle$, acquired at different times within a single period. The excitation frequency was $0.95 \mathrm{GHz}$ and no static external magnetic field was applied. From the normalized $\mathrm{M}_{\mathrm{y}}$ contrast, it is possible to observe spin-waves, confined at the domain wall, propagating away from the antenna (yellow dashed line in the bottom of first snapshot) for more than $2 \mu \mathrm{m}$. The profile extracted at $\mathrm{y}=1.1 \mu \mathrm{m}$ from 
the microstrip antenna (panel b) shows that the spin-waves lateral extension (FWHM) is $150 \mathrm{~nm}$, compatible with the domain wall width ${ }^{23}$, confirming that the mode is confined at the wall.

\section{CONCLUSIONS}

In this, work we show that via tam-SPL it is possible to pattern with nanoscale resolution spin textures in an exchange bias system. This opens the way to a plethora of new applications, from nanoplasmonics to spintronics. In particular, here we demonstrate that reconfigurable nanopatterned spin textures can be used to manipulate spin-waves. By $\mu$ BLS and STXM measurements, we show that spin-waves can be confined in micron-sized 90 deg domains and nano-sized $180 \mathrm{deg}$ domains walls patterned via tam-SPL. While they are localized in the transverse direction, these modes are able to efficiently propagate along the patterned micro- and nano-channels for distance of several microns without the application of external magnetic fields. These results pave the way to the use of tam-SPL for the realization of integrated nanomagnonic computing devices based on spin textures.

\section{ACKNOWLEDGMENTS}

The research leading to these results has received funding from the European Union's Horizon 2020 research and innovation program under grant agreements no. 705326, project SWING, and no. 730872, project CALIPSOplus. We acknowledge the support from the Office of Basic Energy Sciences of the US Department of Energy (grant no. DESC0016204), the US Army Research Laboratory and the US Army Research Office (grant no. W911NF-16-1-0113). This work was partially performed at Polifab, the micro- and nano-technology center of the Politecnico di Milano. Part of this work was performed at the PolLux (X07DA) endstation of the Swiss Light Source, Paul Scherrer Institut, Villigen, Switzerland. The PolLux endstation was financed by the German Minister für Bildung und Forschung (BMBF) through contracts $05 \mathrm{KS} 4 \mathrm{WE} 1 / 6$ and $05 \mathrm{KS} 7 \mathrm{WE} 1$.

\section{REFERENCES}

[1] Neusser, S. and Grundler, D., "Magnonics: Spin Waves on the Nanoscale,” Adv. Mater. 21(28), 2927-2932 (2009).

[2] Lenk, B., Ulrichs, H., Garbs, F., Münzenberg, M. and Münzenberg, M., "The building blocks of magnonics," Phys. Rep. 507(4-5), 107-136 (2011).

[3] Karenowska, A. D., Chumak, A. V., Serga, A. A. and Hillebrands, B., "Magnon spintronics," Handb. Spintron. 11(June), 1505-1549 (2015).

[4] Csaba, G., Papp, Á. and Porod, W., "Perspectives of using spin waves for computing and signal processing," Phys. Lett. A 381(17), 1471-1476 (2017).

[5] Kruglyak, V. V, Demokritov, S. O. and Grundler, D., "Magnonics,” J. Phys. D. Appl. Phys. 43(26), 264001 (2010).

[6] Haldar, A., Kumar, D. and Adeyeye, A. O., "A reconfigurable waveguide for energy-efficient transmission and local manipulation of information in a nanomagnetic device," Nat. Nanotechnol. 11(5), 437-443 (2016).

[7] Krawczyk, M. and Grundler, D., "Review and prospects of magnonic crystals and devices with reprogrammable band structure," J. Phys. Condens. Matter 26(12), 123202 (2014).

[8] Vogt, K., Schultheiss, H., Jain, S., Pearson, J. E., Hoffmann, A., Bader, S. D. and Hillebrands, B., "Spin waves turning a corner," Appl. Phys. Lett. 101(4) (2012).

[9] Stigloher, J., Decker, M., Körner, H. S., Tanabe, K., Moriyama, T., Taniguchi, T., Hata, H., Madami, M., Gubbiotti, G., Kobayashi, K., Ono, T. and Back, C. H., "Snell's Law for Spin Waves," Phys. Rev. Lett. 117(3), 
$1-5$ (2016).

[10] Vogt, K., Fradin, F. Y., Pearson, J. E., Sebastian, T., Bader, S. D., Hillebrands, B., Hoffmann, A. and Schultheiss, H., "Realization of a spin-wave multiplexer.," Nat. Commun. 5, 3727 (2014).

[11] Garcia-Sanchez, F., Borys, P., Soucaille, R., Adam, J. P., Stamps, R. L. and Kim, J. Von., "Narrow Magnonic Waveguides Based on Domain Walls," Phys. Rev. Lett. 114(24), 1-5 (2015).

[12] Winter, J. M., "Bloch wall excitation. Application to nuclear resonance in a bloch wall," Phys. Rev. 124(2), 452459 (1961).

[13] Wagner, K., Kákay, A., Schultheiss, K., Henschke, A., Sebastian, T. and Schultheiss, H., "Magnetic domain walls as reconfigurable spin-wave nanochannels," Nat. Nanotechnol. 11(5), 432-436 (2016).

[14] Albisetti, E., Petti, D., Pancaldi, M., Madami, M., Tacchi, S., Curtis, J., King, W. P., Papp, A., Csaba, G., Porod, W., Vavassori, P., Riedo, E. and Bertacco, R., "Nanopatterning reconfigurable magnetic landscapes via thermally assisted scanning probe lithography," Nat. Nanotechnol. 11(6), 545-551 (2016).

[15] Albisetti, E., Petti, D., Madami, M., Tacchi, S., Vavassori, P., Riedo, E. and Bertacco, R., "Nanopatterning spintextures: A route to reconfigurable magnonics," AIP Adv. 7(5) (2017).

[16] Albisetti, E., Caló, A., Spieser, M., Knoll, A. W., Riedo, E. and Petti, D., "Stabilization and control of topological magnetic solitons via nanoscale patterning of the exchange bias," submitted.

[17] Sharma, P. P., Albisetti, E., Monticelli, M., Bertacco, R. and Petti, D., "Exchange bias tuning for magnetoresistive sensors by inclusion of non-magnetic impurities," Sensors (Switzerland) 16(7) (2016).

[18] Petti, D., Albisetti, E., Reichlová, H., Gazquez, J., Varela, M., Molina-Ruiz, M., Lopeandía, A. F., Olejník, K., Novák, V., Fina, I., Dkhil, B., Hayakawa, J., Marti, X., Wunderlich, J., Jungwirth, T. and Bertacco, R., "Storing magnetic information in IrMn/MgO/Ta tunnel junctions via field-cooling," Appl. Phys. Lett. 102(19) (2013).

[19] Garcia, R., Knoll, A. W. and Riedo, E., "Advanced scanning probe lithography,” Nat. Nanotechnol. 9(8), 577587 (2014).

[20] Albisetti, E., Carroll, K. M., Lu, X., Curtis, J. E., Petti, D., Bertacco, R. and Riedo, E., "Thermochemical scanning probe lithography of protein gradients at the nanoscale," Nanotechnology 27(31) (2016).

[21] Donahue, M. J. and Porter, D. G., [OOMMF User's Guide, Version 1.0. Interagency Report NISTIR 6376] (2004).

[22] Vansteenkiste, A., Leliaert, J., Dvornik, M., Helsen, M., Garcia-Sanchez, F. and Van Waeyenberge, B., "The design and verification of MuMax3," AIP Adv. 4(10), 107133 (2014).

[23] Albisetti, E. and Petti, D., "Domain wall engineering through exchange bias," J. Magn. Magn. Mater. 400(February), 230-235 (2016).

[24] Madami, M., Gubbiotti, G., Tacchi, S. and Carlotti, G., "Application of Microfocused Brillouin Light Scattering to the Study of Spin Waves in Low-Dimensional Magnetic Systems," [Solid State Physics, vol.63], R. E. C. and R. L. Stamps, Ed., Burlington: Academic Press, 79-150 (2012).

[25] Albisetti, E., Petti, D., Sala, G., Silvani, R., Tacchi, S., Finizio, S., Wintz, S., Caló, A., Zheng, X., Raabe, J., Riedo, E. and Bertacco, R., "Nanomagnonic waveguides based on reconfigurable spin-textures for spin computing," arXiv:1712.08293 (2017).

[26] Raabe, J., Tzvetkov, G., Flechsig, U., Böge, M., Jaggi, A., Sarafimov, B., Vernooij, M. G. C., Huthwelker, T., Ade, H., Kilcoyne, D., Tyliszczak, T., Fink, R. H. and Quitmann, C., "PolLux: A new facility for soft X-ray spectromicroscopy at the swiss light source," Rev. Sci. Instrum. 79(11), 1-10 (2008). 